\title{
EUSO-SPB2 Telescope Optics and Testing
}

\author{
Viktoria Kungel, ${ }^{a, *}$ Randy Bachman, ${ }^{a}$ Jerod Brewster, ${ }^{a}$ Madeline Dawes, ${ }^{a}$ Julianna \\ Desiato, ${ }^{a}$ Johannes Eser, ${ }^{b}$ William Finch, ${ }^{a}$ Lindsey Huelett, ${ }^{a}$ Angela V. Olinto, ${ }^{b}$ \\ Justin Pace, ${ }^{a}$ Miroslav Pech, ${ }^{c}$ Patrick Reardon, ${ }^{d}$ Petr Schovanek, ${ }^{c}$ Chantal Wang ${ }^{a}$ \\ and Lawrence Wiencke ${ }^{a}$ on behalf of the JEM-EUSO Collaboration \\ (a complete list of authors can be found at the end of the proceedings) \\ ${ }^{a}$ Colorado School of Mines, \\ Golden, CO, USA \\ ${ }^{b}$ University of Chicago, \\ Chicago, IL, USA \\ ${ }^{c}$ Joint Laboratory of Optics, Palacky University and Institute of Physics AV $\check{C} R$, \\ Olomouc, Czech Republic \\ ${ }^{d}$ The University of Alabama in Huntsville, \\ Huntsville, IL, USA \\ E-mail: kungel@mines . edu
}

The Extreme Universe Space Observatory - Super Pressure Balloon (EUSO-SPB2) mission will fly two custom telescopes that feature Schmidt optics to measure Čerenkov- and fluorescence-emission of extensive air-showers from cosmic rays at the PeV and EeV-scale, and search for $\tau$-neutrinos. Both telescopes have 1-meter diameter apertures and UV/UV-visible sensitivity. The Čerenkov telescope uses a bifocal mirror segment alignment, to distinguish between a direct cosmic ray that hits the camera versus the Čerenkov light from outside the telescope. Telescope integration and laboratory calibration will be performed in Colorado. To estimate the point spread function and efficiency of the integrated telescopes, a test beam system that delivers a 1-meter diameter parallel beam of light is being fabricated. End-to-end tests of the fully integrated instruments will be carried out in a field campaign at dark sites in the Utah desert using cosmic rays, stars, and artificial light sources. Laser tracks have long been used to characterize the performance of fluorescence detectors in the field. For EUSO-SPB2 an improvement in the method that includes a correction for aerosol attenuation is anticipated by using a bi-dynamic Lidar configuration in which both the laser and the telescope are steerable. We plan to conduct these field tests in Fall 2021 and Spring 2022 to accommodate the scheduled launch of EUSO-SPB2 in 2023 from Wanaka, New Zealand.

$37^{\text {th }}$ International Cosmic Ray Conference (ICRC 2021)

July 12th - 23rd, 2021

Online - Berlin, Germany

\footnotetext{
*Presenter
} 


\section{Mission}

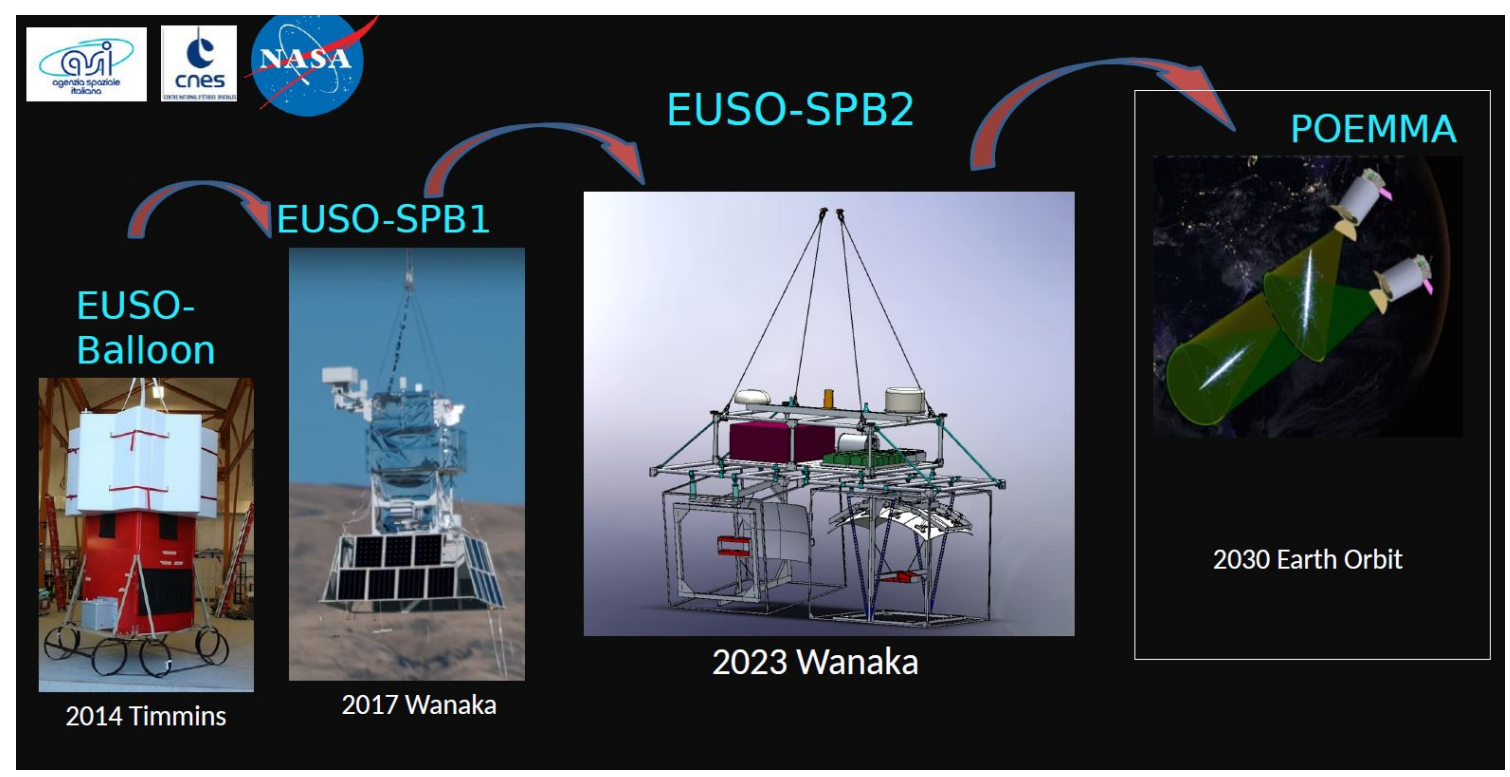

Figure 1: EUSO-SPB2 is a scientific and technological pathfinder for space based astroparticle experiments. It's missions objective is to measure UHECR with the fluorescence and direct Čerenkov-technique from the stratosphere and search for high energy cosmic neutrinos. The design is based on the experience gained in precursors like EUSO-Balloon [1], launched 2014 and EUSO-SPB2 [2], launched 2017.

The Extreme Universe Space Observatory - Super Pressure Balloon (EUSO-SPB2) [3] is a cosmic ray detector, acting as a payload on board of a NASA super pressure balloon. EUSO-SPB2 is primarily a technological and scientific pathfinder, see Fig. 1 and proof of concept for future space experiments, including POEMMA (Probe Of Multi-Messenger Astrophysics) [4]. Going to space has the advantage that a large area of the sky can be covered and observed, to compensate the very small flux and rare occurrence of ultra high energy cosmic rays (UHECR). EUSO-SPB2 will have the first observation of UHECRs from near-orbit altitude with the fluorescence- and directČerenkov-technique at $33 \mathrm{~km}$ or $<7$ mbar altitude. Below the limb it will search for tau neutrinos $v_{\tau}$ [5]. Target launch is April 2023 from Wanaka, New Zealand, with an anticipated flight duration of 100 days.

\section{EUSO-SPB2 detector}

The hardware consists of two custom telescopes that measure Čerenkov- and fluorescenceemission from extensive air-showers at the PeV and EeV-scale. The fluorescence telescope camera consists of 3 Photo Detector Modules (PDMs) made of Multi Anode Photomultiplier Tubes (MAPMT, Hamamatsu) with a total of 6912 pixel. The instruments wide field of view (FoV) is $3 \times(11 \times 11)^{\circ}$. A BG3 UV-transmitting band pass filter placed on top of the cameras focal surface gives a wavelength sensitivity of $300-420 \mathrm{~nm}$. The time resolution is $1000 \mathrm{~ns} / \mathrm{GTU}$, where GTU is the gate time unit. The fluorescence telescope will be in nadir position to look for the signature of 
Table 1: The EUSO-SPB2 hardware specification for both telescopes.

\begin{tabular}{|l||l|l|}
\hline \multicolumn{2}{|c|}{ EUSO-SPB2 specs } \\
\hline Telescopes & Fluorescence FT & Čerenkov CE \\
\hline Wavelength Sensitivity & UV 300-420 nm & no filter $(300-900 \mathrm{~nm})$ \\
Energy Threshold & EeV & PeV \\
Sensor Type & MAPMT (Hamamatsu) & SiPM $(\mathrm{S} 14521-6050 \mathrm{CN})$ \\
Field of View & $3 \times(11 \times 11)^{\circ}$ & $(6.4 \times 12.8)^{\circ}$ \\
Pixel FOV & $(0.2 \times 0.2)^{\circ}$ & $(0.4 \times 0.4)^{\circ}$ \\
Time frame & $1000 \mathrm{~ns} / \mathrm{bin}$ & $10 \mathrm{~ns}$ \\
Pointing (zenith angle) & nadir & Earth's limb \pm 10 \\
\hline
\end{tabular}

extensive air showers (EAS) of UHECR, i.e. the fluorescence photon scattering from the electromagnetic component.

The Čerenkov telescope camera consists of one camera made of Silicon Photomultiplier (SiPM, Hamamatsu (S14521-6050CN)) with 512 pixel. The instruments FoV is $6.4^{\circ} \times 12.8^{\circ}$, so the primary mirror can be smaller in size. The wavelength sensitivity is UV-visible with $300-900 \mathrm{~nm}$. The Črenkov telescope is a high speed detector with a time sensitivity of $10 \mathrm{~ns}$. It tilts $\pm 10^{\circ}$ to look towards the Earth's limb to see the emission of EAS produced by high energy $v_{\tau}$. Both telescopes have an achromatic PMMA corrector plate at the 1-m diameter aperture, which is UV transparent. [6].

\section{Optics}

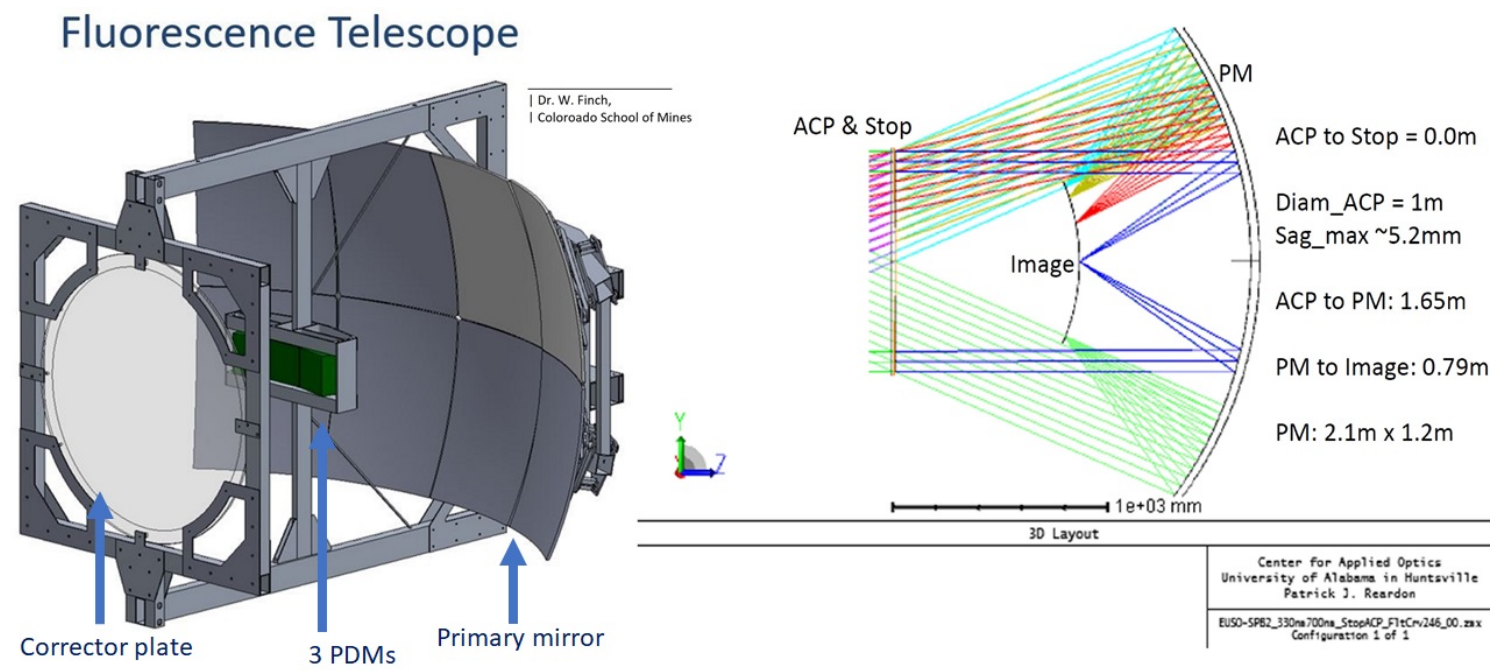

Figure 2: The fluorescence telescope design on the left and the corresponding raytracing model on the right. You can see the primary mirror PM, the achromatic corrector plate ACP with its diameter and maximum sag, as well as different distances between the components.

The telescopes feature modified Schmidt optics [7], with spherical borosilicate mirror glass. 
The current design anticipates 6 mirror segments for the fluorescence and 4 mirror segments for the Čerenkov telescope, where both have a radius of curvature $\mathrm{ROC}=1659.8 \mathrm{~mm}$ with an effective focal length $\mathrm{EFL}=860 \mathrm{~mm}$. For the Čerenkov telescope a bi-focal mirror is used. One mirror in a row is tilted relative to the other one around vertical axis, with the optical axis is in horizontal position as seen in Fig. 2. This creates a second separated spot at the focal surface, to distinguish between a charged particle which makes one flash on the camera and a light pulse from far-field outside the telescope, which makes two spots. The ratio of the collection area from the upper and lower mirrors depends on the direction of the incoming light, and affects the ratio of flux in the bifocal spots. The fluorescence telescope is designed to measure track like signals. Its 3 PDMs form a curved image surface, so that a field correcting or flattening lens is necessary to achieve a very small spot. The light reflected by the primary mirror propagates through a plano-convex lens and a BG3 filter before it hits the elementary cell and the PMTS. Without taking the BG3 filter into account, the overall throughput estimation, including corrector plate, mirror and filter losses is around 0.67 .

On the back side the mirror segments of each telescope are assembled by a newly designed and fabricated holding structure as seen in Fig. 3. Whippletrees hold around $20 \mathrm{lbs}$. mirror segments on nine attachment points that are glued to a bonding disk on spherical bearings. The bonding disks are made of Kovar, which has approximately the same thermal expansion as glass. This design provides stability and high alignment precision, however, with an equally distributed force that orients the bond disks according the curvature of the mirror without bending it.

\section{Mirror Segment Assembly}
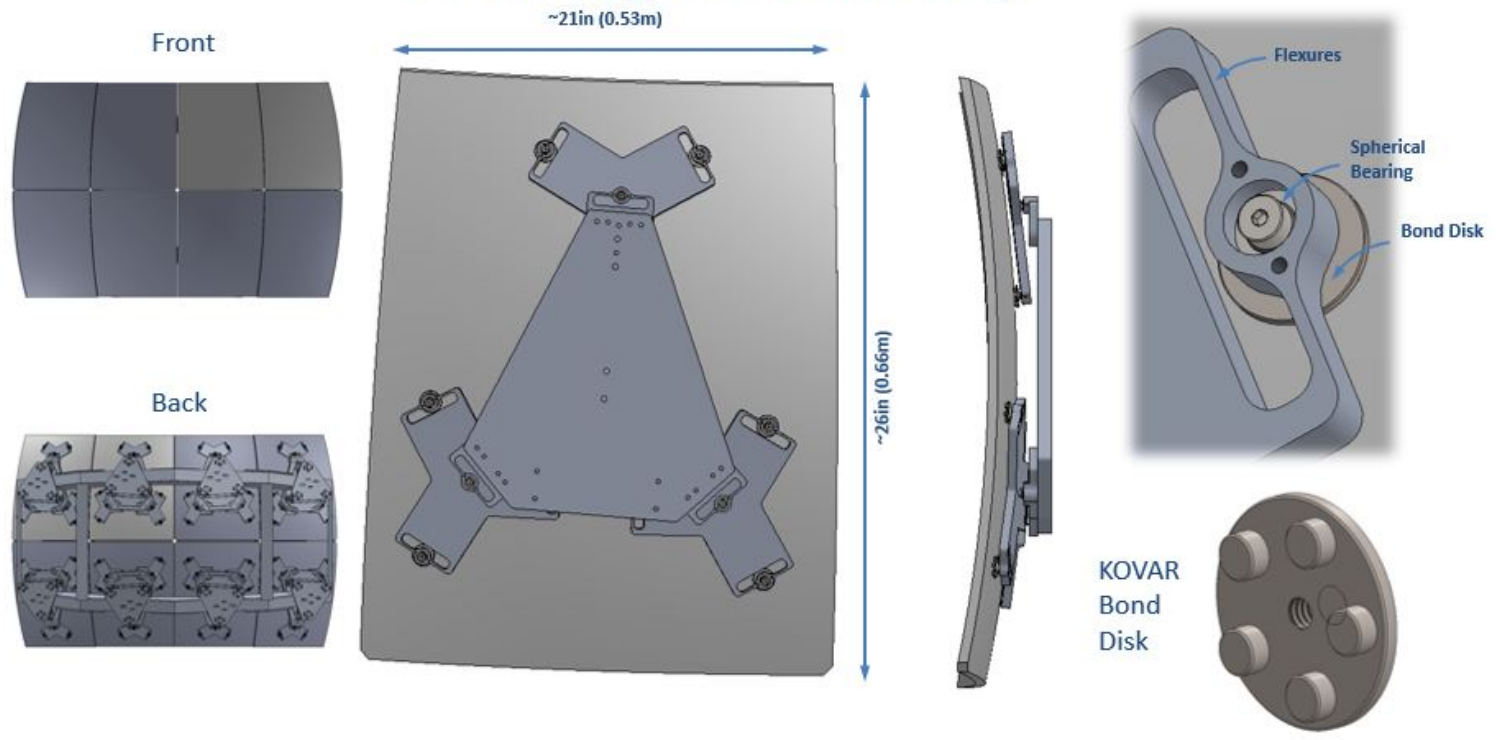

Figure 3: Mirror segment assembly with customized mirror segment attachment. The glass is epoxy glued to Kovar bonding disks. Kovar is a nickel-cobalt ferrous alloy and has approximately the same thermal expansion as glass. 


\section{Testing}

EUSO-SPB2 tests do verify design specifications that have to meet scientific and technological objectives. EUSO-SPB2 is a stratospheric experiment and differs mainly from ground based experiments in terms of its accessibility. The design requirements are considerable more stringent, for example it has to meet thermal and mechanical requirements. Additionally, flight hardware has redundancies, to reduce the risk of failure and ensure the missions success. However, the main goals of the laboratory tests carried out at Colorado school of Mines are the determination of the point spread function (PSF) and efficiency of both telescopes, via a fabricated 1-m parallel test beam system, seen in Fig. 4.

The idea is that LED emitted light for different UV-range wavelengths $(340,365,385 \mathrm{~nm})$ is

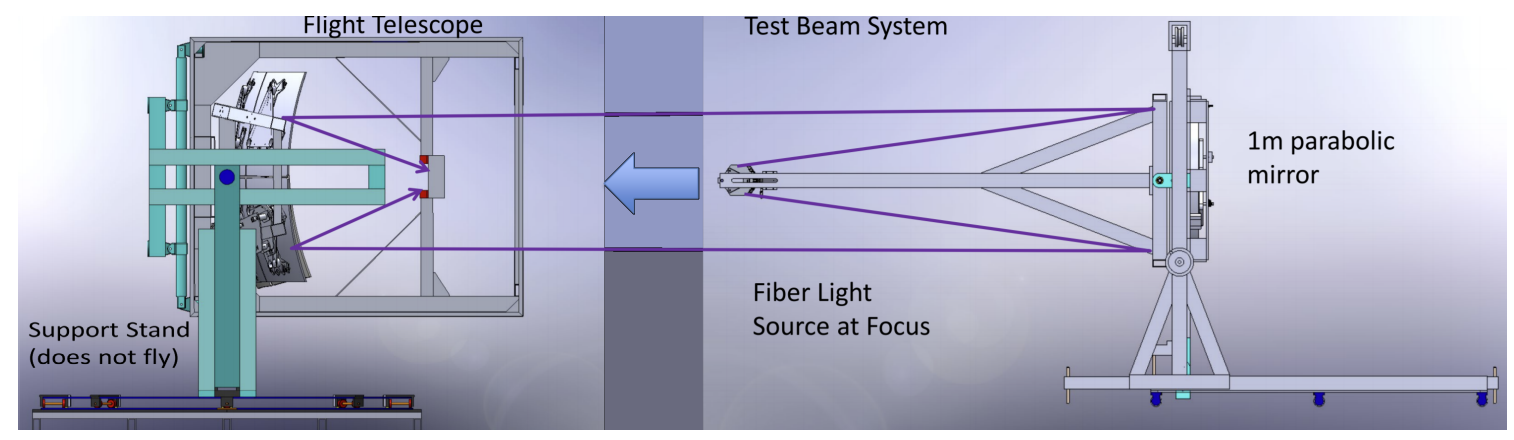

Figure 4: A schematic configuration of the fluorescence telescope on a supporting stand on the left and a 1-m diameter test beam system on the right. The test beam system is used to illuminate the telescope evenly with parallel light beam in the UV-light range. The main goal is to determine the PSF and efficiency.

reflected on a UV enhanced coated 1-m diameter parabolic F3.5 mirror, that provides a parallel beam illuminating the 1-m diameter aperture of the telescope. The lights intensity will then be measured with the help of a diode, that will be placed directly at the aperture on a rotating stage, symmetrical as a windmill. The stage will be operated remotely by stepper motors and a lead screw for a stable measurement where the step width and velocity is regulated. Afterwards, the intensity will be measured in the area where around $95 \%$ of the light accumulates, without the camera integrated. For this a Cartesian robot system, again driven by stepper motors, will be used inside the mounting plates of the camera. Finally, the camera is mounted and read out, with intensity and PSF being determined.

To make sure there is no displacement of the mirror segment, and thus a distortion of the spot size, the mirror alignment and its systems are tested in the lab. There is no option to correct a shift or a degree of freedom in the mounting system while flight. The mirror segments are epoxy glued to the metallic frame with EC-2216 adhesive, that is known for its high peel and shear strength, and durability over huge temperature cycles. The glued system is also tested as part of the operational design requirements of NASA. The glue joints are stress tested under a static uniformly distributed $20 \mathrm{G}$ load, in a thermal chamber for temperature below $-40^{\circ} \mathrm{C}$, and in a low pressure environment of $<7$ mbar, to simulate atmospheric conditions, see Fig. 5 for an example of a glue joint being under $43 \mathrm{lbs}$ of stress. Before operating the telescopes the radius of curvature, with all mounted mirror segments will be confirmed. 

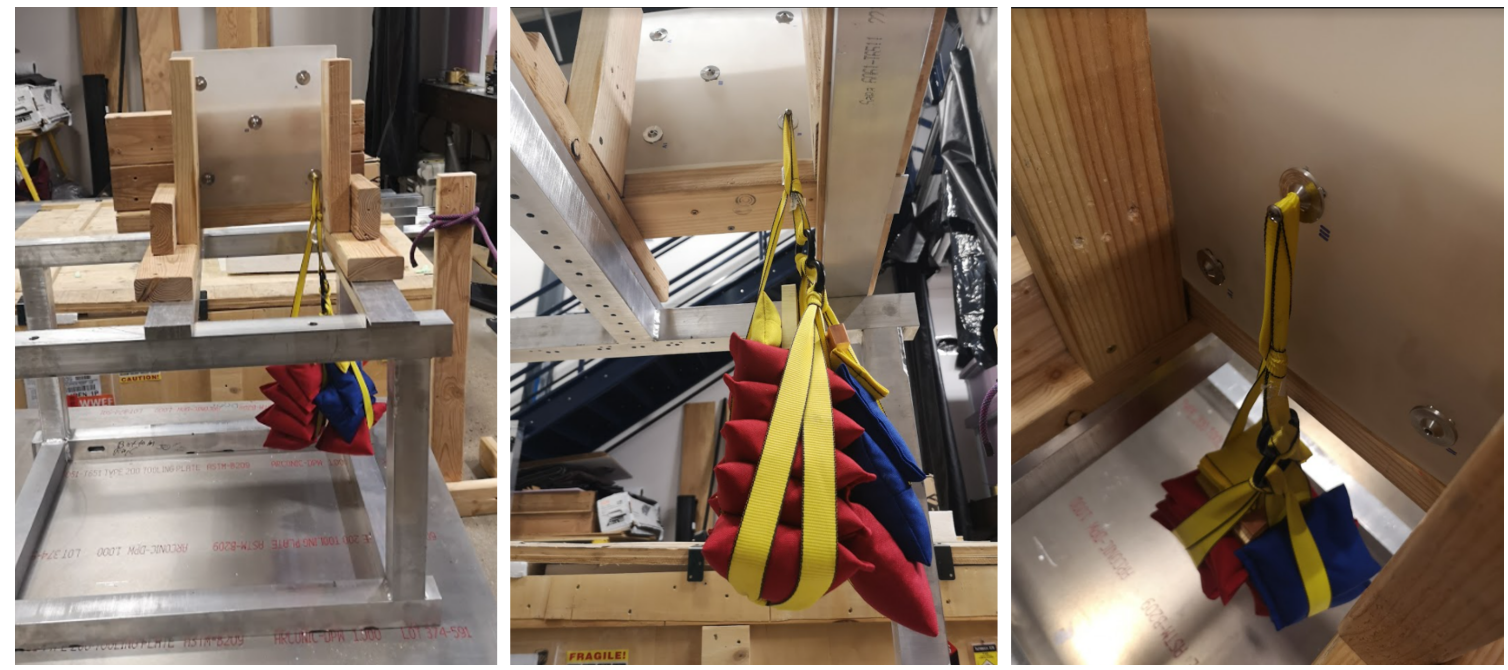

Figure 5: Test stand in shear or Čerenkov configuration. The Kovar bond disks are epoxy glued to curved test glass piece, that has the same surface roughness as the primary mirror. The adhesive is under $43 \mathrm{lbs}$. shear stress with a small proportion of peeling stress in ambient. The test was successful and no break was recorded. Continuing test in various thermal and pressure conditions are currently under processing

\section{Field campaign}

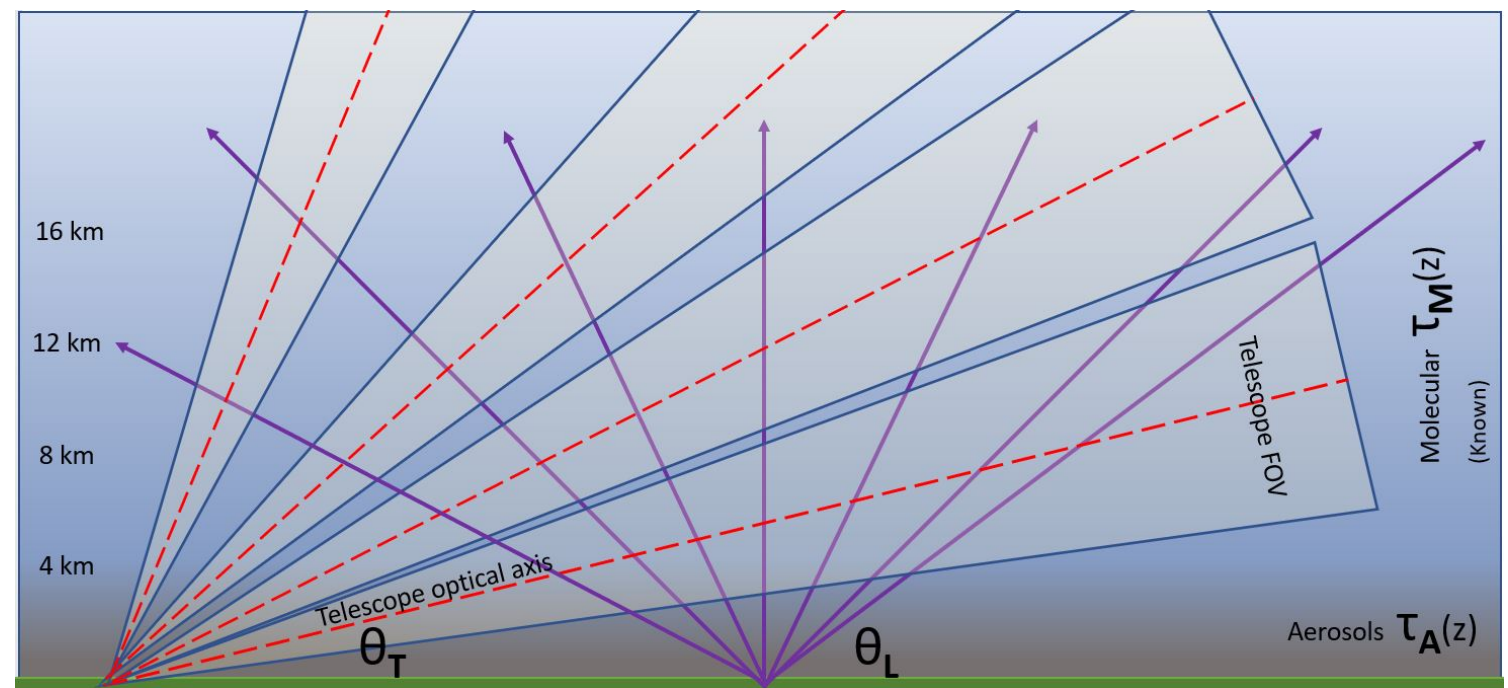

Figure 6: Sketch of the the field test configuration with the steerable telescope on the left and the steerable laser on the right with different inclination angles $\theta_{T}$ and $\theta_{L}$. The laser beam crosses the telescopes field of view high above the aerosol layer.

Field tests will be performed in the desolate dark sites in the Western USA, in Utah, to check the detectors performance at moonless nights. The main goal of the field campaign is to determine the field of view and the energy trigger threshold for both telescopes. The campaign will include an absolute photometric calibration of the fully integrated instruments with the help of a bi-dynamic Lidar configuration, Fig 6. The laser system is inside a mobile utility trailer and can be leveled, 
so that the optical enclosure and the periscope is decoupled from the driving rack, when aiming in all directions above horizon. It will be possible to estimate the aerosol optical depth, without using the absolute calibration of the fluorescence telescope as a constraint, by steering the laser and the telescope to collect data over a range of distances and angles. From this an absolute photometric calibration of the fluorescence telescope can be made knowing the laser energy. This newly developed calibration method with a correction for aerosol attenuation will be tested first time. Adopted Monte Carlo laser simulations have been made to predict the detectors performance, find a correlation and parametrization between laser energies and UHECR energies.

Acknowledgment: This work was partially supported by Basic Science Interdisciplinary Research Projects of RIKEN and JSPS KAKENHI Grant (22340063, 23340081, and 24244042), by the Italian Ministry of Foreign Affairs and International Cooperation, by the Italian Space Agency through the ASI INFN agreements n. 2017-8-H.0 and n. 2021-8-HH.0, by NASA award 11-APRA-0058, 16-APROBES16-0023, 17-APRA17-0066, NNX17AJ82G, NNX13AH54G, 80NSSC18K0246, 80NSSC18K0473, 80NSSC19K0626, and 80NSSC18K0464 in the USA, by the French space agency CNES, by the Deutsches Zentrum für Luft- und Raumfahrt, the Helmholtz Alliance for Astroparticle Physics funded by the Initiative and Networking Fund of the Helmholtz Association (Germany), by Slovak Academy of Sciences MVTS JEM-EUSO, by National Science Centre in Poland grants 2017/27/B/ST9/02162 and 2020/37/B/ST9/01821, by Deutsche Forschungsgemeinschaft (DFG, German Research Foundation) under Germany's Excellence Strategy - EXC-2094-390783311, by Mexican funding agencies PAPIIT-UNAM, CONACyT and the Mexican Space Agency (AEM), as well as VEGA grant agency project 2/0132/17, and by by State Space Corporation ROSCOSMOS and the Interdisciplinary Scientific and Educational School of Moscow University "Fundamental and Applied Space Research".

\section{References}

[1] G. Abdellaoui et al. Ultra-violet imaging of the night-time earth by euso-balloon towards space-based ultra-high energy cosmic ray observations. Astroparticle Physics, 111:54-71, 2019.

[2] J. Eser. Results of the EUSO-SPB1 flight. PoS, ICRC2019:247, 2019.

[3] L. Wiencke et al. The Extreme Universe Space Observatory on a Super-Pressure Balloon II Mission. PoS, ICRC2019:466, 2019.

[4] A.V. Olinto et al. The POEMMA (probe of extreme multi-messenger astrophysics) observatory. Journal of Cosmology and Astroparticle Physics, 2021(06):007, jun 2021.

[5] A. L. Cummings, R. Aloisio, and J. F. Krizmanic. Modeling of the tau and muon neutrinoinduced optical cherenkov signals from upward-moving extensive air showers. Physical Review $D, 103(4)$, Feb 2021.

[6] A. Zuccaro Marchi, Y. Takizawa, and Y. Takahashi. The JEM-EUSO optics design. In 32nd International Cosmic Ray Conference, 2011.

[7] V. Da Deppo, P. Sandri, P. Mazzinghi, and A. Zuccaro Marchi. A lightweight Schmidt space telescope configuration for ultra-high energy cosmic ray detection. In Zoran Sodnik, Nikos Karafolas, and Bruno Cugny, editors, International Conference on Space Optics - ICSO 2018, volume 11180, pages 2527 - 2537. International Society for Optics and Photonics, SPIE, 2019. 


\section{Full Authors List: JEM-EUSO Collaboration}

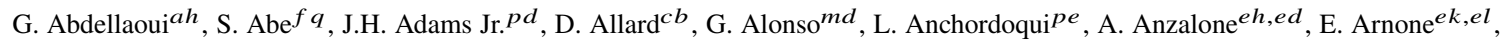

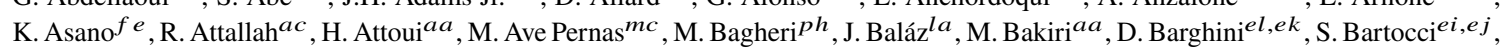
M. Battisti ${ }^{e k, e l}$, J. Bayer ${ }^{d d}$, B. Beldjilaliah ${ }^{a}$ T. Belenguer ${ }^{m b}$, N. Belkhalfa ${ }^{a a}$, R. Bellotti ${ }^{e a, e b}$, A.A. Belov ${ }^{k b}$, K. Benmessai ${ }^{a a}$, M. Bertaina ${ }^{e k, e l}$, P.F. Bertone ${ }^{p f}$, P.L. Biermann ${ }^{d b}$, F. Bisconti ${ }^{e l, e k}$, C. Blaksley ${ }^{f t}$, N. Blanc ${ }^{o a}$, S. Blin-Bondil $^{c a, c b}$, P. Bobik ${ }^{l a}$, M. Bogomilov $b a$, K. Bolmgren ${ }^{n a}$, E. Bozzo ${ }^{o b}$, S. Briz ${ }^{p b}$, A. Bruno ${ }^{e h, e d}$, K.S. Caballero ${ }^{h d}$, F. Cafagna ${ }^{e a}$, G. Cambiéei,ej,

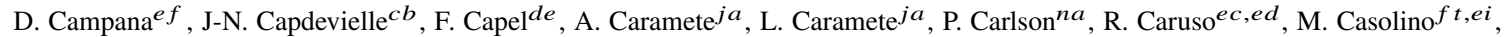

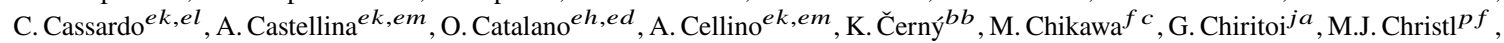
R. Colalillo ${ }^{e f}, e g$, L. Conti ${ }^{e n, e i}$, G. Cotto ${ }^{e k, e l}$, H.J. Crawford ${ }^{p a}$, R. Cremonini ${ }^{e l}$, A. Creusot ${ }^{c b}$, A. de Castro Gónzalez ${ }^{p b}$, C. de la Taille ${ }^{c a}$, L. del Peral ${ }^{m c}$, A. Diaz Damian ${ }^{c c}$, R. Diesing ${ }^{p b}$, P. Dinaucourt ${ }^{c a}$, A. Djakonow ${ }^{i a}$, T. Djemil ${ }^{a c}$, A. Ebersoldt ${ }^{d b}$, T. Ebisuzaki $^{f t}$, J. Eser $^{p b}$, F. Fenu ${ }^{e k, e l}$, S. Fernández-González ${ }^{m a}$, S. Ferrarese $e^{e k, e l}$, G. Filippatos ${ }^{p c}$, W.I. Finch $^{p c}$ C. Fornaro ${ }^{e n, e i}$, M. Fouka ${ }^{a b}$, A. Franceschie ${ }^{e e}$ S. Franchini ${ }^{m d}$, C. Fuglesang ${ }^{n a}$, T. Fujii ${ }^{f g}$, M. Fukushima ${ }^{f e}$, P. Galeotti $^{e k, e l}$, E. García-Ortega $^{m a}$, D. Gardiole ${ }^{e k, e m}$, G.K. Garipov ${ }^{k b}$, E. Gascón ${ }^{m a}$, E. Gazda $^{p h}$, J. Genci $^{l b}$, A. Golzio ${ }^{e k, e l}$, C. González Alvarado ${ }^{m b}$, P. Gorodetzky $^{f t}$, A. Green ${ }^{p c}$, F. Guarino $e f, e g$, C. Guépin ${ }^{p l}$, A. Guzmán ${ }^{d d}$, Y. Hachisu ${ }^{f t}$, A. Haungs $d b$, J. Hernández Carretero ${ }^{m c}$, L. Hulett $^{p c}$,

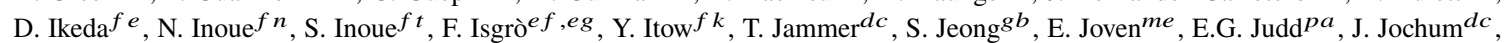
F. Kajino $f f$, T. Kajino ${ }^{f i}$, S. Kalli ${ }^{a f}$, I. Kaneko ${ }^{f t}$, Y. Karadzhov $b a$, M. Kasztelan ${ }^{i a}$, K. Katahira ${ }^{f t}$, K. Kawai ${ }^{f t}$, Y. Kawasaki ${ }^{f t}$, A. Kedadra ${ }^{a a}$, H. Khales ${ }^{a a}$, B.A. Khrenov ${ }^{k b}$, Jeong-Sook Kim ${ }^{g a}$, Soon-Wook Kim ${ }^{g a}$, M. Kleifges ${ }^{d b}$, P.A. Klimov ${ }^{k b}$, D. Kolev ${ }^{b a}$,

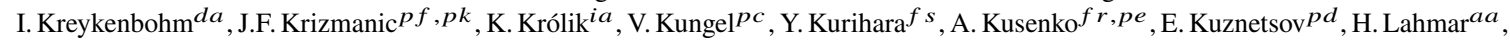
F. Lakhdari ${ }^{a g}$, J. Licandro ${ }^{m e}$, L. López Campano ${ }^{m a}$, F. López Martínez ${ }^{p b}$, S. Mackovjak $^{l a}$, M. Mahdi $^{a a}$, D. Mandát ${ }^{b c}$, M. Manfrin $^{e k, e l}$,

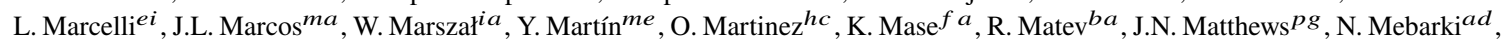

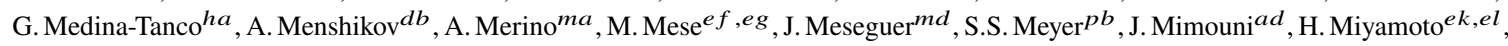
Y. Mizumoto $f i$, A. Monaco $e a, e b$, J.A. Morales de los Ríos ${ }^{m c}$, M. Mastafa ${ }^{p d}$, S. Nagataki ${ }^{f t}$, S. Naitamor ${ }^{a b}$, T. Napolitano $^{e e}$, J. M. Nachtman ${ }^{p i}$ A. Neronov $a b, c b$, K. Nomoto $f r$, T. Nonaka ${ }^{f e}$, T. Ogawa ${ }^{f t}$, S. Ogio $f l$, H. Ohmori ${ }^{f t}$, A.V. Olinto $^{p b}$, Y. Onel $^{p i}$

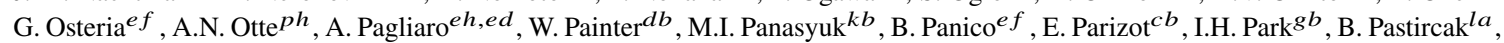
T. Paul ${ }^{p e}$, M. Pech ${ }^{b b}$, I. Pérez-Grande ${ }^{m d}$, F. Perfetto ${ }^{e f}$, T. Peter ${ }^{o c}$, P. Picozza ${ }^{e i, e j, f t}$, S. Pindado ${ }^{m d}$, L.W. Piotrowski ${ }^{i b}$, S. Piraino ${ }^{d d}$, Z. Plebaniak $e^{e k, e l, i a}$, A. Pollinioa , E.M. Popescu ${ }^{j a}$, R. Prevete ${ }^{e f, e g}$, G. Prévôt ${ }^{c b}$, H. Prieto ${ }^{m c}$, M. Przybylak ${ }^{i a}$, G. Puehlhofer ${ }^{d d}$, M. Putis ${ }^{l a}$, P. Reardon ${ }^{p d}$, M.H.. Reno ${ }^{p i}$, M. Reyes ${ }^{m e}$, M. Ricciee ${ }^{e}$ M.D. Rodríguez Frías ${ }^{m c}$, O.F. Romero Matamala $^{p h}$, F. Ronga ${ }^{e e}$, M.D. Sabau ${ }^{m b}$, G. Saccáec,ed , G. Sáez Cano ${ }^{m c}$, H. Sagawa ${ }^{f e}$, Z. Sahnoune ${ }^{a b}$, A. Saito $^{f g}$, N. Sakaki ${ }^{f t}$, H. Salazar $^{h c}$, J.C. Sanchez Balanzar ${ }^{h a}$, J.L. Sánchez ${ }^{m a}$, A. Santangelo ${ }^{d d}$, A. Sanz-Andrés ${ }^{m d}$, M. Sanz Palomino ${ }^{m b}$, O.A. Saprykin ${ }^{k c}$, F. Sarazin ${ }^{p c}$, M. Sato ${ }^{f o}$, A. Scagliola $e a, e b$, T. Schanz ${ }^{d d}$, H. Schieler ${ }^{d b}$, P. Schovánek ${ }^{b c}$, V. Scotti ${ }^{e f}, e g$, M. Serra $^{m e}$, S.A. Sharakin ${ }^{k b}$, H.M. Shimizu $f j$, K. Shinozaki ${ }^{i a}$, J.F. Soriano ${ }^{p e}$, A. Sotgiu ${ }^{e i, e j}$, I. Stan ${ }^{j a}$, I. Strharskýla, N. Sugiyama ${ }^{f j}$, D. Supanitsky ${ }^{h a}$,

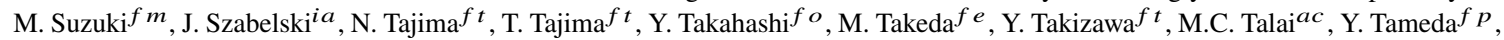

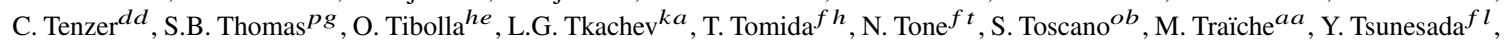
K. Tsuno ${ }^{f t}$, S. Turriziani ${ }^{f t}$, Y. Uchihori $f b$, O. Vaduvescume ${ }^{m}$ J.F. Valdés-Galicia ${ }^{h a}$, P. Vallania ${ }^{e k, e m}$, L. Valore ${ }^{e f}, e g$, G. VankovaKirilova $^{b a}$, T. M. Venters ${ }^{p j}$, C. Vigorito ${ }^{e k, e l}$, L. Villaseñor ${ }^{h b}$, B. Vlcek ${ }^{m c}$, P. von Ballmoos ${ }^{c c}$, M. Vrabel ${ }^{l b}$, S. Wada ${ }^{f t}$, J. Watanabe ${ }^{f i}$, J. Watts Jr. ${ }^{p d}$, R. Weigand Muñoz ${ }^{m a}$, A. Weindl ${ }^{d b}$, L. Wiencke ${ }^{p c}$, M. Wille ${ }^{d a}, \mathrm{~J}$. Wilms ${ }^{d a}$, D. Winn ${ }^{p m}$ T. Yamamoto $f f$, J. Yang ${ }^{g b}$, H. Yano $f m$, I.V. Yashin ${ }^{k b}$, D. Yonetoku $f d$, S. Yoshida $f a$, R. Young Y $^{p f}$, I.S Zgura ${ }^{j a}$, M.Yu. Zotov $k b$, A. Zuccaro Marchif $f$

aa Centre for Development of Advanced Technologies (CDTA), Algiers, Algeria

$a b$ Dep. Astronomy, Centre Res. Astronomy, Astrophysics and Geophysics (CRAAG), Algiers, Algeria

${ }^{a c}$ LPR at Dept. of Physics, Faculty of Sciences, University Badji Mokhtar, Annaba, Algeria

ad Lab. of Math. and Sub-Atomic Phys. (LPMPS), Univ. Constantine I, Constantine, Algeria

af Department of Physics, Faculty of Sciences, University of M'sila, M'sila, Algeria

ag Research Unit on Optics and Photonics, UROP-CDTA, Sétif, Algeria

$a h$ Telecom Lab., Faculty of Technology, University Abou Bekr Belkaid, Tlemcen, Algeria

ba St. Kliment Ohridski University of Sofia, Bulgaria

$b b$ Joint Laboratory of Optics, Faculty of Science, Palacký University, Olomouc, Czech Republic

$b c$ Institute of Physics of the Czech Academy of Sciences, Prague, Czech Republic

ca Omega, Ecole Polytechnique, CNRS/IN2P3, Palaiseau, France

$c b$ Université de Paris, CNRS, AstroParticule et Cosmologie, F-75013 Paris, France

$c c$ IRAP, Université de Toulouse, CNRS, Toulouse, France

da ECAP, University of Erlangen-Nuremberg, Germany

$d b$ Karlsruhe Institute of Technology (KIT), Germany

$d c$ Experimental Physics Institute, Kepler Center, University of Tübingen, Germany

$d d$ Institute for Astronomy and Astrophysics, Kepler Center, University of Tübingen, Germany

de Technical University of Munich, Munich, Germany

$e a$ Istituto Nazionale di Fisica Nucleare - Sezione di Bari, Italy

$e b$ Universita' degli Studi di Bari Aldo Moro and INFN - Sezione di Bari, Italy

ec Dipartimento di Fisica e Astronomia "Ettore Majorana", Universita' di Catania, Italy 
$e^{e d}$ Istituto Nazionale di Fisica Nucleare - Sezione di Catania, Italy

$e e$ Istituto Nazionale di Fisica Nucleare - Laboratori Nazionali di Frascati, Italy

ef Istituto Nazionale di Fisica Nucleare - Sezione di Napoli, Italy

eg Universita' di Napoli Federico II - Dipartimento di Fisica "Ettore Pancini", Italy

eh INAF - Istituto di Astrofisica Spaziale e Fisica Cosmica di Palermo, Italy

${ }^{e i}$ Istituto Nazionale di Fisica Nucleare - Sezione di Roma Tor Vergata, Italy

$e^{e j}$ Universita' di Roma Tor Vergata - Dipartimento di Fisica, Roma, Italy

${ }^{e k}$ Istituto Nazionale di Fisica Nucleare - Sezione di Torino, Italy

el Dipartimento di Fisica, Universita' di Torino, Italy

em Osservatorio Astrofisico di Torino, Istituto Nazionale di Astrofisica, Italy

en Uninettuno University, Rome, Italy

$f a$ Chiba University, Chiba, Japan

$f b$ National Institutes for Quantum and Radiological Science and Technology (QST), Chiba, Japan

$f c$ Kindai University, Higashi-Osaka, Japan

$f d$ Kanazawa University, Kanazawa, Japan

$f e$ Institute for Cosmic Ray Research, University of Tokyo, Kashiwa, Japan

$f f$ Konan University, Kobe, Japan

$f g$ Kyoto University, Kyoto, Japan

$f^{h}$ Shinshu University, Nagano, Japan

$f^{i}$ National Astronomical Observatory, Mitaka, Japan

$f j$ Nagoya University, Nagoya, Japan

${ }^{f k}$ Institute for Space-Earth Environmental Research, Nagoya University, Nagoya, Japan

${ }^{f l}$ Graduate School of Science, Osaka City University, Japan

${ }^{f m}$ Institute of Space and Astronautical Science/JAXA, Sagamihara, Japan

${ }^{f n}$ Saitama University, Saitama, Japan

$f o$ Hokkaido University, Sapporo, Japan

$f p$ Osaka Electro-Communication University, Neyagawa, Japan

$f q$ Nihon University Chiyoda, Tokyo, Japan

${ }^{f r}$ University of Tokyo, Tokyo, Japan

$f s$ High Energy Accelerator Research Organization (KEK), Tsukuba, Japan

${ }^{f t}$ RIKEN, Wako, Japan

ga Korea Astronomy and Space Science Institute (KASI), Daejeon, Republic of Korea

${ }^{g b}$ Sungkyunkwan University, Seoul, Republic of Korea

ha Universidad Nacional Autónoma de México (UNAM), Mexico

${ }^{h b}$ Universidad Michoacana de San Nicolas de Hidalgo (UMSNH), Morelia, Mexico

${ }^{h c}$ Benemérita Universidad Autónoma de Puebla (BUAP), Mexico

hd Universidad Autónoma de Chiapas (UNACH), Chiapas, Mexico

he Centro Mesoamericano de Física Teórica (MCTP), Mexico

ia National Centre for Nuclear Research, Lodz, Poland

${ }^{i b}$ Faculty of Physics, University of Warsaw, Poland

$j a$ Institute of Space Science ISS, Magurele, Romania

$k a$ Joint Institute for Nuclear Research, Dubna, Russia

${ }^{k b}$ Skobeltsyn Institute of Nuclear Physics, Lomonosov Moscow State University, Russia

${ }^{k c}$ Space Regatta Consortium, Korolev, Russia

la Institute of Experimental Physics, Kosice, Slovakia

$l b$ Technical University Kosice (TUKE), Kosice, Slovakia

ma Universidad de León (ULE), León, Spain

${ }^{m b}$ Instituto Nacional de Técnica Aeroespacial (INTA), Madrid, Spain

$m c$ Universidad de Alcalá (UAH), Madrid, Spain

${ }^{m d}$ Universidad Politécnia de madrid (UPM), Madrid, Spain

me Instituto de Astrofísica de Canarias (IAC), Tenerife, Spain

${ }^{n a}$ KTH Royal Institute of Technology, Stockholm, Sweden

oa Swiss Center for Electronics and Microtechnology (CSEM), Neuchâtel, Switzerland

${ }^{o b}$ ISDC Data Centre for Astrophysics, Versoix, Switzerland

${ }^{o c}$ Institute for Atmospheric and Climate Science, ETH Zürich, Switzerland

$p^{a}$ Space Science Laboratory, University of California, Berkeley, CA, USA

$p^{b}$ University of Chicago, IL, USA

$p c$ Colorado School of Mines, Golden, CO, USA

${ }^{p d}$ University of Alabama in Huntsville, Huntsville, AL; USA

$p e$ Lehman College, City University of New York (CUNY), NY, USA

$p f$ NASA Marshall Space Flight Center, Huntsville, AL, USA 
$p g$ University of Utah, Salt Lake City, UT, USA

$p^{h}$ Georgia Institute of Technology, USA

${ }^{p i}$ University of Iowa, Iowa City, IA, USA

${ }^{p j}$ NASA Goddard Space Flight Center, Greenbelt, MD, USA

${ }^{p k}$ Center for Space Science \& Technology, University of Maryland, Baltimore County, Baltimore, MD, USA

${ }^{p l}$ Department of Astronomy, University of Maryland, College Park, MD, USA

${ }^{p m}$ Fairfield University, Fairfield, CT, USA 